eine mehr an den Lehrer sich wendende Buchserie zu erkennen. Das schließt keineswegs aus, daß es nicht auch dem Schüler der Mittelschulstufe verständlich sei. Just der Verfasser von Band I hat es sich angelegen sein lassen, den S:off in leicht falslicher Art darzustellen, wobei auch berücksichtigt wurde, daß das Lehrwerk über die Schule hinaus einem weitern Kreise dienen soll und kann. Er hat dabei vor allem den Wissensstoff der Physiogeographie eingehend verarbeitet. Damit gab er der Kenntnis des Kulturcharakters der verschiedenen Länder eine solide Grundlage, während er die "Anthropogeographie“ selbst bewußt zurücktreten ließ. Ob dies, wie das Vorwort betont, durch die Tatsache allein gerechtfertigt ist, daß in den letzten Jahren „derart umfangreiche Umsiedlungen innerhalb der einzelnen Länder .. stattgefunden (haben), daß es noch geraumer Zeit bedarf, bis wieder Zustände von Dauer vorliegen werden“, kann in Frage gezogen werden. Die geographische "Substanz" ist schon seit Jahrzehnten infolge Industrialisierung und Technisierung so tief und stetig in Umschichtung gegriffen, daß auch ohne Kriege und deren Folgen „Zustände von Dauer" kaum zu erwarten sind. Andrerseits liegt gerade im Landschaftswandel doch eine der reizvollsten Aufgaben für den Geographie-Unterricht. Der Autor hat sich selbst schon dadurch korrigiert, daß er zahlreiche anthropogeographische Bemerkungen in die Physiogeographie der Länder flocht und hat zudem auch besondere wirtschaftliche, siedlungskundliche und politische Kapitel geschrieben, die mindestens den Rahmen anthropogeographischer Betrachtungen umreißen. Die Gesamtdarstellung gliedert sich einleuchtend in die Kapitel Nordeuropa, Mitteleuropa, Osteuropa (worunter die europäischen Teile der UdSSR und Rumänien verstanden sind, eine Zuteilung, die besonders deswegen erwägenswert ist, weil die UdSSR heute kaum mehr - wenn schon nach politischen Einheiten dargestellt wird - auseinandergerissen werden dürfte), die in sich in begrüßenswerte Gesamtübersichten und erklärende Beschreibungen der einzelnen Staaten zerfallen. Innerhalb dieser Abschnitte wird nach dem für viele Fälle bewährten sngenannten länderkundlichen Schema verfahren, wobei jedoch keineswegs stets die gleiche Reihenfolge der Landschaftsfaktoren eingehalten ist. Vielmehr werden Dominanten herausgearbeitet, häufig ist auch an die Regionen der Länder angeknüpft (Norwegen : Südküste, Oslogebiet, Westküste, Südliches Hochland, Drontheimergebiet, Nordland, Fischerei, Tromsö, Finnmark, Staat und Wirtschaft), woıaus sich der Eindruck freier Gestaltung der Materie ergibt. Es war an sich schon immer sehr schwer, aus dem Riesenstoff das "Wesentliche“ für eine bestimmte Schulstufe ausz"'wählen; das zeigt ein Vergleich mit der bereits wieder vorhandenen Reilie teils ausgezeichneter deutscher Lehrbücher, die für das Lehrwerk sowohl einen instruktiven Maßstab als auch einen Ansporn zu erfolgreichem Wettbewerb darstellen.

Noch schwieriger als die textliche Bewältigung des länderkundlichen Stoffes erscheint es, die wichtigsten Landschaftstypen der dargestellten Regionen in relativ wenigen, charakteristischen Bildern festzuhalten. Dies ist $\mathrm{m}$. E. dem Autor sehr gut gelungen, wenn auch zu wünschen wäre, daß die 48 Typenbilder im Texte verteilt ständen. Dem Verlag ist nahezulegen, daß er bei kommenden Bänden zwischen Text und Bild keine Verlagsanzeigen einschiebt. Lohensivert ist im ganzen der einfache klare Stil des mit zahlreichen Kartenskizzen unterstützten Textes. Im übrigen wird eine eingehendere Würdigung erst vorgenommen werden können, wenn andere Bände des Gesamtwerkes Vergleiche und Beurteilung der Absichten der Herausgeber detaillierter zulassen. Nicht zuletzt in diesem Zusammenhange ist zu hoffen, daß die Fortsetzung bald folgen werde und daß die kommenden Bände die Erwartungen rechtfertigen, die die schweizerischen Geographielehrer - aller Stufen - auf sie setzen.

W. KÜNDIG-STEINER

\title{
UNE NOUVELLE CARTE ECONOMIQUE DE L'EUROPE
}

Publiée à Stockholm en 1953 par Generalstabens Litografiska Anstalts Förlag, cette carte au 3250000 e est l'œuvre commune de nombreux géographes européens. Nous la devons à l'initiative de Monsieur W. Wir.r.IAm-Oısson, professeur de géographie économique à Stockholm, qui signe comme éditeur. La tâche des auteurs n'était pas facile. Il fallait, en effet, rapporter au même dénominateur des statistiques souvent basées sur des points de vue divergeants. Je me bornerai à donner ici un aperçu sommaire des signes conventionnels. Pour de plus amples détails le lecteur voudra bien consulter la brochure de 12 pages qui accompagne la carte. Les mers sont de couleurs bleues, les terres de couleurs grises. Le gris foncé indique les espaces se trouvant au dessus de la limite de la forêt ainsi que la toundra, le gris pâle les étendues non cultivées. De petits carrés blancs, dont le côté correspond selon l'échelle à $10 \mathrm{~km}$., représentent des surfaces cultivées de $100 \mathrm{~km}^{2}$. Les branches d'activité sont divisées en 1) agriculture, sylviculture et pêche 2) industrie et artisanat 3) autres occupations (commerce, communications, services publics, administration, hôtellerie, enseignement, etc.). La carte ne nous parle que des agglomérations de plus de 10000 habitants. Comme en général la population agricole ne se concentre pas dans de si grandes agglomérations et que les carrés mentionnés plus haut ne nous font rien savoir sur l'intensité et la nature des cultures, on peut dire que l'agriculture a été plutôt négligée, sans doute par manque de données. De petits cercles blancs indiquent les villages. Plus de $50 \%$ de leur population active est occupée dans l'agriculture, la sylviculture et la pêche. Les villages-villes, représentés par des cercles blancs aux contours renforcés, sont des agglomérations dont le nombre des personnes actives employées dans l'agriculture, la sylviculture et la pêche varie entre 25 et $50 \%$. Les villes sont 
divisées en villes de service et villes industrielles. Les unes comme les autres abritent une population active dont plus de $75 \%$ vit des occupations énumérées sous les rubriques 2 et 3 . Elles ont pour signes conventionnels des sphères munies d'un réseau de coordonnées dont le volume correspond au nombre d'habitants. Leur ombre, projetée sur la carte par un faisceau lumineux se trouvant dans le secteur nord-ouest, n'a aucune signification. Dans les villes de service aux sphères noires, le nombre des employés (numéro 3) dépasse celui des ouvriers (numéro 2) et dans les villes industrielles aux sphères de couleurs, c'est l'inverse. Parmi les villes industrielles la légende distingue d'abord des villes industrielles simples où plus de $50 \%$ des ouvriers travaillent dans une seule industrie, soit dans l'industrie du métal et de l'extraction de minerais, soit dans l'industrie de la houille et du pétrole, soit dans l'industrie des textiles, de l'habillement et des chaussures, soit dans l'industrie du bois et du papier, soit enfin dans une autre branche indépendante, ensuite des villes industrielles doubles où plus de $66,7 \%$ des ouvriers sont occupés dans 2 branches de l'industrie et enfin des villes industrielles multiples où aucune branche ne prédomine. Chacune des branches dans laquelle la population ouvrière d'une ville industrielle simple est employée a une couleur différente. Toutes les villes industrielles doubles n'ayant qu'une seule couleur, il est impossible d'apprendre à quelles branches appartiennent les 2 industries les plus importantes. Deux cartes spéciales à échelle plus grande nous permettent d'obtenir des renseignements détaillés sur les régions industrielles d'Angleterre d'une part et les régions industrielles du nord de la France, de Belgique, des Pays-Bas et de l'Allemagne occidentale d'autre part.

Pour terminer, il faut signaler un fait qui prête à l'équivoque : le texte explicatif a pour point de départ la population exerçant une activité professionelle, la légende, par contre, ne parle que de la population en général. Malgré les observations faites, il faut saluer cette nouvelle tentative de reproduire par la carte l'économie européenne.

R. MEIER

\section{STATUTEN}

\section{DES VERBANDES SCHWEIZERISCHER GEOGRAPHISCHER GESELLSCHAFTEN}

\section{Name, Sitz und Zweck}

Art. I. Die Geographischen Gesellschaften der Schweiz, welche die vorliegenden Statuten anerkannt haben, bilden den Verband Schweizerischer Geographischer Grsellschaften (VSGG), Fédération des Sociétés Suisses de Géographie, Federazione delle Società Svizzere di Geografia, Uniun da las Societads Svizzras da Geografia.

Art. 2. Der Sitz des Verbandes befindet sich am jeweiligen Sitz des Zentralvorstandes.

Art. 3. Der VSGG bezweckt die Förderung der Geographie und ihre Anwendung zum Wohle des Vaterlandes.

Art. 4. Der VSGG sucht diese Zwecke zu erreichen durch:

a) Zusammenschluß aller Geographischen Gesellschaften der Schweiz als sogenannte Zweiggesellschaften zu Unternehmungen, die eines allgemeinen Zusammenwirkens bedürfen ;

b) Förderung der Tätigkeit der bestehenden und Gründung von weiteren Geographischen Gesellschaften;

c) Abhaltung von Exkursionen und Versammlungen mit wissenschaftlichen Vorträgen und Mitteilungen, abwechslungsweise an verschiedenen Orten des Landes in Verbindung mit den Zweiggesellschaften;

d) Ernennung von Kommissionen zur Ausführung bestimmter Aufgaben :

e) Herausgabe von wissenschaftlichen Veröffentlichungen;

f) Unterstützung wissenschaftlicher Forschungen, insbesondere auch von Forschungsreisen ;

g) Förderung der Landschaftsplanung, sowie landschaftsgestaltender und landschaftsschützender Maßnahmen;

h) Förderung der Beziehungen zu Nachbarwissenschaften und deren Organisationen; der VSGG ist Mitglied der Schweizerischen Naturforschenden Gesellschaft (S.N.G.), und organisiert an deren Jahreszusammenkunft jeweils die Sitzungen der „Sektion für Geographie und Kartographie“;

i) Mitwirkung an internationalen geographischen Bestrebungen;

k) Vertretung der schweizerischen Geographie in internationalen Vereinigungen (Int. Geographische Union, UNESCO usw.) und an Kongressen.

\section{Mitglieder}

Art. 5. Die Aufnahme einer $Z$ weiggesellschaft wird auf Grund ihres Gesuches, dem die Statuten beizulegen sind, von der Delegierten-Versammlung vollzogen. 